\section{Warnhinweise für strahleninduzierte Ösophagitis}

\begin{abstract}
Bei Patienten, die wegen eines nichtkleinzelligen Lungenkarzinoms (NSCLC) bestrahlt werden, kann es zur strahleninduzierten Ösophagitis kommen. Nun ging man der Frage nach, welche Faktoren das Risiko dafür erhöhen.
\end{abstract}

\begin{abstract}
A uf der Suche nach patienten- und therapiebezogenen Faktoren, die bei der Vorhersage einer strahleninduzierten Ösophagitis helfen könnten, analysierte man die Daten von 652 NSCLCPatienten. Alle Patientne hatten sich einer Strahlentherapie ihres Lungenkarzinoms unterzogen: 405 einer 3D-konformalen Strahlentherapie (3D-CRT), 139 einer intensitätsmodulierten $\mathrm{Be}$ strahlung (IMRT) und 108 einer Protonenstrahltherapie (PBT). Für alle waren volle Dosis-Volumen-Histogramme verfügbar.

Eine schwere strahleninduzierte Ösophagitis ( $\geq \operatorname{Grad} 3$ ) war am häufigsten in der IMRT-Gruppe: mit $28 \%$ bei signifikant mehr Patienten als nach 3D-CRT $(8 \%)$ und PBT (6\%). Vom Beginn der Strahlentherapie bis zum Auftreten
\end{abstract}

schwerer Strahlenösophagitiden vergingen median 42 Tage (11-93 Tage). Ein Großteil machte sich bereits vor Abschluss der Bestrahlungen bemerkbar.

Die Analyse dosimetrischer Faktoren mit dem Lyman-Kutcher-Burman (LKB)-Modell ergab, dass sich die fraktionierte effektive Dosis signifikant von der fraktionierten mittleren Dosis unterschied. Für die Vorhersage einer schweren strahleninduzierten Ösophagitis war vor allem die hohe Strahlendosis auf kleines Volumen von Bedeutung, weniger die mittlere Ösophagusdosis. Patienten, die mehr als 30 Fraktionen erhielten, waren häufiger betroffen. Als einziger klinischer bzw. therapiebezogener Faktor, der eine Ösophagitis $\geq$, Grad 3 vorhersagte, erwies sich eine gleichzeitige Chemotherapie. Gerade der Einsatz von Taxanen schien sich in dieser Hinsicht ungünstig auszuwirken.

In dem auf Dosis-Volumen-Histogrammen, Zahl der Fraktionen und gleichzeitiger Chemotherapie basierenden Risikomodell wurde die Inzidenz schwerer Strahlenösophagitiden bei den mit IMRT behandelten Patienten unterschätzt. Auch bei Berücksichtigung dosimetrischer und klinischer Faktoren erwies sich gerade diese Methode als Risikofaktor für die Ösphagitisentwicklung.

Fazit: Die Autoren empfehlen, bei der NSCLC-Strahlentherapie nicht nur die mittlere Ösophagusdosis ( $\leq 34 \mathrm{~Gy}$ ) zu beachten, sondern auch das mit $\geq 70$ Gy bestrahlte Volumen der Speiseröhre auf $<20 \%$ zu beschränken. Die maximale Ösophagusdosis sollte 80 Gy nicht überschreiten.

Ulrike Wepner

Gomez DR et al. Predictors of high-grade esophagitis after definitive three-dimensional conformal therapy, intensity-modulated radiation therapy, or proton beam therapy for nonsmall cell lung cancer. Int J Radiat Oncol Biol Phys. 2012;84(4):1010-6.

\title{
Trio gegen Cisplatin-induziertes Erbrechen
}

\begin{abstract}
Patienten, die eine hoch emetogene Chemotherapie mit Cisplatin über fünf Tage erhalten, benötigen eine potente Prophylaxe gegen Übelkeit und Erbrechen. In einer Crossover-Studie stand die Kombination von einem 5-HT3-Antagonisten und Dexamethason mit dem Neurokinin-1-Rezeptorantagonisten Aprepitant auf dem Prüfstand.
\end{abstract}

$B^{c}$ ei Patienten, die eintägig mit Cisplatin-Kombination behandelt werden, hat sich zur antiemetischen Prophylaxe Aprepitant zusätzlich zu einem 5-HT3Antagonisten und Dexamethason bewährt. Damit lässt sich auch das verzögerte Auftreten von Übelkeit und Erbrechen wirksam bekämpfen. Ein Problem stellt die mehrtägige Anwendung hoch emetogener Chemotherapeutika wie Cisplatin dar, da den Patienten verzögerte Nausea und Emesis vom Behandlungsbeginn und Akutsymptome der Folgetage gleichzeitig zu schaffen machen. In einer randomisierten, placebokontrollierten, doppelblinden Crossover-Studie wurde nun das prophylaktische Potenzial von Aprepitant in dieser Situation geprüft.
69 Patienten, die wegen eines Keimzelltumors eine jeweils fünftägige Cisplatin-basierte Chemotherapie erhielten, bekamen im ersten Zyklus zusätzlich zur Standardprophylaxe mit einem 5-HT3Antagonisten (Tag 1-5) und Dexamethason (je $20 \mathrm{mg}$ an Tag 1 und Tag 2) randomisiert entweder Aprepitant (125 mg an Tag 3, $80 \mathrm{mg} / \mathrm{d}$ an den Tagen 4-7) oder Placebo. Im zweiten Zyklus wechselten sie zum jeweils anderen Regime.

$42 \%$ der Patienten mit Aprepitant zeigten ein komplettes Ansprechen kein Erbrechen und keine Rescue-Therapie -, $13 \%$ mit Placebo ( $<<0,001) .11 \mathrm{~Pa}-$ tienten (16,2\%) im Aprepitant-Zyklus hatten mindestens eine emetische Episode, 32 (47,1\%) im Placebo-Zyklus. 38
Patienten bevorzugten Aprepitant, 11 Placebo ( $\mathrm{p}<0,001)$. Beim Erfassen der Übelkeit auf einer visuellen Analogskala fand sich zwar kein statistisch signifikanter Unterschied, doch schnitt Aprepitant numerisch besser ab als Placebo. Eine vermehrte Toxizität war unter Aprepitant nicht zu beobachten.

Fazit: Patienten, die eine hoch emetogene Chemotherapie mit Cisplatin über fünf Tage erhielten, profitierten von der Zugabe des NK-1-Rezeptorantagonisten Aprepitant zu einem 5-HT3-Antagonisten und Dexamethason. Ein komplettes Ansprechen war signifikant häufiger als mit Placebo. Kein Wunder, dass die Patienten diese Form der antiemetischen Prophylaxe bevorzugten. Ulrike Wepner

Albany $C$ et al. Randomized, double-blind, placebo-controlled, phase III cross-over study evaluating the oral neurokinin-1 antagonist aprepitant in combination with a $5 \mathrm{HT} 3$ receptor antagonist and dexamethasone in patients with germ cell tumors receiving 5-day cisplatin combination chemotherapy regimens: a Hoosier Oncology Group study. J Clin Oncol. 2012;30(32):3998-4003. 